\title{
Design Artificial Intelligence-Based Switching PD plus Gravity for Highly Nonlinear Second Order System
}

\author{
Farzin Piltan $^{\mathrm{a}}$, Mahdi Jafari ${ }^{\mathrm{b}}$, Mehdi Eram ${ }^{\mathrm{b}}$, Omid Mahmoudi $^{\mathrm{b}}$, Omid Reza Sadrnia ${ }^{\mathrm{b}}$ \\ ${ }^{a}$ Senior Researcher at Research and Development Department of SanatkadeheSabze Pasargad Company \\ (S.S.P. Co), Shiraz, Iran \\ ${ }^{b}$ Research and Development Unit, SanatkadeheSabze Pasargad Company (S.S.P. Co), Shiraz, Iran
}

\begin{abstract}
Refer to this research, an intelligent fuzzy parallel switching Proportional-Derivative (PD) plus gravity controller is proposed for highly nonlinear continuum robot manipulator. Design a nonlinear controller for second order nonlinear uncertain dynamical systems is one of the most important challenging works. In order to provide high performance in nonlinear systems, switching partly sliding mode plus gravity controller is selected. Pure switching partly sliding mode plus gravity controller can be used to control of partly known nonlinear dynamic parameters of continuum robot manipulator. Conversely, this method is used in many applications; it must to solve chattering phenomenon which it can cause some problems such as saturation and heat the mechanical parts of continuum robot manipulators or drivers. In order to solve the chattering phenomenon, implement easily and avoid mathematical model base controller, Mamdani's performance/errorbased fuzzy logic methodology with two inputs and one output and 49 rules is parallel applied to pure switching partly sliding mode plus gravity controller. The results demonstrate that this method is a model-free controllers which works well in certain and partly uncertain system.
\end{abstract}

Index Terms: Fuzzy inference system, Mamdani fuzzy inference engine, sliding mode control, continuum robot, PD plus gravity.

(C) 2013 Published by MECS Publisher. Selection and/or peer review under responsibility of the Research Association of Modern Education and Computer Science.

\section{Introduction}

The international organization defines the robot as "an automatically controlled, reprogrammable, multipurpose manipulator with three or more axes." Robot manipulator is a collection of links that connect to each other by joints, these joints can be revolute and prismatic that revolute joint has rotary motion around an axis and prismatic joint has linear motion around an axis. Each joint provides one or more degrees of freedom

* Corresponding author.

E-mail address: ${ }^{* 1}$ piltan_f@iranssp.com; ${ }^{* 2}$ ssp.robotic@gmail.com 
(DOF). From the mechanical point of view, robot manipulator is divided into two main groups, which called; serial robot links and parallel robot links. In serial robot manipulator, links and joints is serially connected between base and final frame (end-effector). Most of continuum robots are serial links, which in $n$ degrees of freedom serial link robot manipulator the axis of the first three joints has a known as major axis, these axes show the position of end-effector, the axis number four to six are the minor axes that use to calculate the orientation of end-effector and the axis number seven to $n$ use to reach the avoid the difficult conditions (e.g., surgical robot and space robot manipulator). Dynamic modeling of continuum robot manipulators is used to describe the behavior of continuum robot manipulator such as linear or nonlinear dynamic behavior, design of model based controller such as pure sliding mode controller and pure computed torque controller which design these controller are based on nonlinear dynamic equations, and for simulation. The dynamic modeling describes the relationship between joint motion, velocity, and accelerations to force/torque or current/voltage and also it can be used to describe the particular dynamic effects (e.g., inertia, coriolios, centrifugal, and the other parameters) to behavior of system[1]. Continuum robots represent a class of robots that have a biologically inspired form characterized by flexible backbones and high degrees-of-freedom structures [1]. The idea of creating "trunk and tentacle" robots, (in recent years termed continuum robots [1]), is not new [2]. Inspired by the bodies of animals such as snakes [3], the arms of octopi [4], and the trunks of elephants [5], [6], researchers have been building prototypes for many years. A key motivation in this research has been to reproduce in robots some of the special qualities of the biological counterparts. This includes the ability to "slither" into tight and congested spaces and (of particular interest in this work) the ability to grasp and manipulate a wide range of objects, via the use of "whole arm manipulation" i.e. wrapping their bodies around objects, conforming to their shape profiles. Hence, these robots have potential applications in whole arm grasping and manipulation in unstructured environments such as rescue operations. Theoretically, the compliant nature of a continuum robot provides infinite degrees of freedom to these devices. However, there is a limitation set by the practical inability to incorporate infinite actuators in the device. Most of these robots are consequently underactuated (in terms of numbers of independent actuators) with respect to their anticipated tasks. In other words they must achieve a wide range of configurations with relatively few control inputs. This is partly due to the desire to keep the body structures (which, unlike in conventional rigid-link manipulators or fingers, are required to directly contact the environment) "clean and soft", but also to exploit the extra control authority available due to the continuum contact conditions with a minimum number of actuators. For example, the Octarm VI continuum manipulator, discussed frequently in this paper, has nine independent actuated degrees-of-freedom with only three sections. Continuum manipulators differ fundamentally from rigid-link and hyper-redundant robots by having an unconventional structure that lacks links and joints. Hence, standard techniques like the Denavit-Hartenberg (D-H) algorithm cannot be directly applied for developing continuum arm kinematics. Moreover, the design of each continuum arm varies with respect to the flexible backbone present in the system, the positioning, type and number of actuators. The constraints imposed by these factors make the set of reachable configurations and nature of movements unique to every continuum robot. This makes it difficult to formulate generalized kinematic or dynamic models for continuum robot hardware. Chirikjian and Burdick were the first to introduce a method for modeling the kinematics of a continuum structure by representing the curve-shaping function using modal functions [6]. Mochiyama used the Serret- Frenet formulae to develop kinematics of hyper-degrees of freedom continuum manipulators [5]. For details on the previously developed and more manipulator-specific kinematics of the Rice/Clemson "Elephant trunk" manipulator, see [1], [2], [5]. For the Air Octor and Octarm continuum robots, more general forward and inverse kinematics have been developed by incorporating the transformations of each section of the manipulator (using D-H parameters of an equivalent virtual rigid link robot) and expressing those in terms of the continuum manipulator section parameters [4]. The net result of the work in [6], [3]-[5] is the establishment of a general set of kinematic algorithms for continuum robots. Thus, the kinematics (i.e. geometry based modeling) of a quite general set of prototypes of continuum manipulators has been developed and basic control strategies now exist based on these. The development of 
analytical models to analyze continuum arm dynamics (i.e. physicsbased models involving forces in addition to geometry) is an active, ongoing research topic in this field. From a practical perspective, the modeling approaches currently available in the literature prove to be very complicated and a dynamic model which could be conveniently implemented in an actual device's real-time controller has not been developed yet. The absence of a computationally tractable dynamic model for these robots also prevents the study of interaction of external forces and the impact of collisions on these continuum structures. This impedes the study and ultimate usage of continuum robots in various practical applications like grasping and manipulation, where impulsive dynamics [1], [4] are important factors. Although continuum robotics is an interesting subclass of robotics with promising applications for the future, from the current state of the literature, this field is still in its stages of inception.

Controller is a device which can sense information from linear or nonlinear system (e.g., continuum robot manipulator) to improve the systems performance [3]. The main targets in designing control systems are stability, good disturbance rejection, and small tracking error[5]. Several continuum robot manipulators are controlled by linear methodologies (e.g., Proportional-Derivative (PD) controller, Proportional- Integral (PI) controller or Proportional- Integral-Derivative (PID) controller), but when continuum robot manipulator works with various payloads and have uncertainty in dynamic models this technique has limitations. In some applications continuum robot manipulators are used in an unknown and unstructured environment, therefore strong mathematical tools used in new control methodologies to design nonlinear robust controller with an acceptable performance (e.g., minimum error, good trajectory, disturbance rejection). Sliding mode controller is an influential nonlinear controller to certain and uncertain systems which it is based on system's dynamic model.

Sliding mode controller is used to control of highly nonlinear systems especially for continuum robot manipulators. The important problem of the pure sliding mode controller with switching function was chattering phenomenon in certain and uncertain systems. In this research the chattering phenomenon problem can be reduced in certain system by using parallel fuzzy logic theory. To eliminate the continuum robot manipulator's dynamic of system and chattering, 49 rules Mamdani inference system is design and applied to PD partly sliding mode plus gravity methodology with switching function. This methodology is worked based on applied fuzzy logic parallel with gravity nonlinear dynamic part to eliminate unknown dynamic parameters.

This paper is organized as follows; section 2, is served as an introduction to the PID controller, feedback linearization compensator, Mamdani fuzzy inference engine to estimate the FLC and its application to control of continuum robot and dynamic of continuum robot. Part 3, introduces and describes the methodology. Section 4 presents the simulation results and discussion of this algorithm applied to a continuum robot and the final section is describing the conclusion.

\section{Theory}

\subsection{Dynamic Formulation of Continuum Robot}

The Continuum section analytical model developed here consists of three modules stacked together in series. In general, the model will be a more precise replication of the behavior of a continuum arm with a greater of modules included in series. However, we will show that three modules effectively represent the dynamic behavior of the hardware, so more complex models are not motivated. The kinetic energy (T) of the system comprises the sum of linear kinetic energy terms (constructed using the above velocities) and rotational kinetic energy terms due to rotation of the rigid rod connecting the two actuators, and is given below as 


$$
\begin{aligned}
\mathrm{T}=(0.5) \mathrm{m}_{1} \dot{\mathrm{s}}_{1}{ }^{2} & +(0.5) \mathrm{m}_{2}\left(\left(\dot{\mathrm{s}}_{2} \sin \theta_{1}+\mathrm{s}_{2} \cos \theta_{1} \dot{\theta}_{1}\right)^{2}+\left(\dot{\mathrm{s}}_{1}+\dot{\mathrm{s}}_{2} \cos \theta_{1}-\mathrm{s}_{2} \sin \theta_{1} \dot{\theta}_{1}\right)^{2}\right) \\
& +(0.5) \mathrm{m}_{3}\left(\left(\dot{\mathrm{s}}_{2} \sin \theta_{1}+\mathrm{s}_{2} \cos \theta_{1} \dot{\theta}_{1}+\dot{\mathrm{s}}_{3} \sin \left(\theta_{1}+\theta_{2}\right)+\mathrm{s}_{3} \cos \left(\theta_{1}+\theta_{2}\right) \dot{\theta}_{1}+\mathrm{s}_{3} \cos \left(\theta_{1}+\theta_{2}\right) \dot{\theta}_{2}\right)^{2}\right. \\
& \left.+\left(\dot{\mathrm{s}}_{1}+\dot{\mathrm{s}}_{2} \cos _{1}-\mathrm{s}_{2} \sin \theta_{1} \dot{\theta}_{1}+\dot{\mathrm{s}}_{3} \cos \left(\theta_{1}+\theta_{2}\right)-\mathrm{s}_{3} \sin \left(\theta_{1}+\theta_{2}\right) \dot{\theta}_{1}-\mathrm{s}_{3} \sin \left(\theta_{1}+\theta_{2}\right) \dot{\theta}_{2}\right)^{2}\right) \\
& +(0.5) \mathrm{I}_{1} \dot{\theta}_{1}{ }^{2}+(0.5) \mathrm{I}_{2}\left(\dot{\theta}_{1}{ }^{2}+\dot{\theta}_{2}{ }^{2}\right) \\
& +(0.5) \mathrm{I}_{3}\left(\dot{\theta}_{1}{ }^{2}+\dot{\theta}_{2}{ }^{2}\right. \\
& \left.+\dot{\theta}_{3}{ }^{2}\right)
\end{aligned}
$$

The potential energy $(\mathrm{P})$ of the system comprises the sum of the gravitational potential energy and the spring potential energy. A small angle assumption is made throughout the derivation. This allows us to directly express the displacement of springs and the velocities associated with dampers in terms of system generalized coordinates.

$$
\begin{array}{rl}
\mathrm{P}=-\mathrm{m}_{1} \mathrm{gs}_{1}-\mathrm{m}_{2} & \mathrm{~g} \\
+ & \left.\mathrm{s}_{1}+\mathrm{s}_{2} \cos \theta_{1}\right)-\mathrm{m}_{3} \mathrm{~g}\left(\mathrm{~s}_{1}+\mathrm{s}_{2} \cos \theta_{1}+\mathrm{s}_{3} \cos \left(\theta_{1}+\theta_{1}\right)\right)+(0.5) \mathrm{k}_{11}\left(\mathrm{~s}_{1}+\left(\frac{1}{2}\right) \theta_{1}-\mathrm{s}_{01}\right)^{2} \\
+ & (0.5) \mathrm{k}_{21}\left(\mathrm{~s}_{1}+(1 / 2) \theta_{1}-\mathrm{s}_{01}\right)^{2}+(0.5) \mathrm{k}_{12}\left(\mathrm{~s}_{2}+(1 / 2) \theta_{2}-\mathrm{s}_{02}\right)^{2} \\
& +(0.5) \mathrm{k}_{22}\left(\mathrm{~s}_{2}+(1 / 2) \theta_{2}-\mathrm{s}_{02}\right)^{2}+(0.5) \mathrm{k}_{13}\left(\mathrm{~s}_{3}+(1 / 2) \theta_{3}-\mathrm{s}_{03}\right)^{2} \\
& +(0.5) \mathrm{k}_{23}\left(\mathrm{~s}_{3}+(1 / 2) \theta_{3}\right. \\
& \left.-\mathrm{s}_{03}\right)^{2}
\end{array}
$$

where, $\mathrm{S}_{01}, \mathrm{~S}_{02}, \mathrm{~S}_{03}$ are the initial values of $\mathrm{S}_{1}, \mathrm{~S}_{2}, \mathrm{~S}_{3}$ respectively.

Due to viscous damping in the system, Rayliegh's dissipation function [6] is used to give damping energy

$$
\begin{aligned}
\mathrm{D}=(0.5) \mathrm{c}_{11}\left(\dot{\mathrm{s}}_{1}\right. & \left.+(1 / 2) \dot{\theta}_{1}\right)^{2}+(0.5) \mathrm{c}_{21}\left(\dot{\mathrm{s}}_{1}+(1 / 2) \dot{\theta}_{1}\right)^{2}+(0.5) \mathrm{c}_{12}\left(\dot{\mathrm{s}}_{2}+(1 / 2) \dot{\theta}_{2}\right)^{2}+(0.5) \mathrm{c}_{22}\left(\dot{\mathrm{s}}_{2}+(1 / 2) \dot{\theta}_{2}\right)^{2} \\
& +(0.5) \mathrm{c}_{13}\left(\dot{\mathrm{s}}_{3}+(1 / 2) \dot{\theta}_{3}\right)^{2} \\
& +(0.5) \mathrm{c}_{23}\left(\dot{\mathrm{s}}_{3}+(1 / 2) \dot{\theta}_{3}\right)^{2}
\end{aligned}
$$

The generalized forces in the system corresponding to the generalized co-ordinates are expressed as appropriately weighted combinations of the input forces.

$$
\begin{aligned}
& Q_{s_{1}}=F_{11}+F_{21}+\left(F_{12}+F_{22}\right) \cos \theta_{1}+\left(F_{13}+F_{23}\right) \cos \left(\theta_{1}+\theta_{2}\right) \\
& Q_{s_{2}}=F_{12}+F_{22}+\left(F_{13}+F_{23}\right) \cos \left(\theta_{2}\right) \\
& Q_{s_{3}}=F_{13}+F_{23} \\
& Q_{\theta_{1}}=(1 / 2)\left(F_{11}-F_{21}\right)+(1 / 2)\left(F_{12}-F_{22}\right)+(1 / 2)\left(F_{13}-F_{23}\right)+s_{2} \sin \theta_{2}\left(F_{13}+F_{23}\right) \\
& Q_{\theta_{1}}=(1 / 2)\left(F_{12}-F_{22}\right)+(1 / 2)\left(F_{13}-F_{23}\right) \\
& Q_{\theta_{1}}=(1 / 2)\left(F_{13}-F_{23}\right)
\end{aligned}
$$


It can be evinced from the force expressions that the total input forces acting on each module can be resolved into an additive component along the direction of extension and a subtractive component that results in a torque. For the first module, there is an additional torque produced by forces in the third module.

The model resulting from the application of Lagrange's equations of motion obtained for this system can be

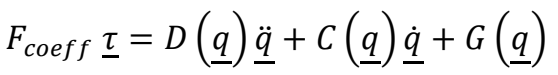

represented in the form

where $\tau$ is a vector of input forces and $\mathrm{q}$ is a vector of generalized co-ordinates. The force coefficient matrix $\mathrm{F}_{\text {coeff }}$ transforms the input forces to the generalized forces and torques in the system. The inertia matrix, $\mathrm{D}$ is composed of four block matrices. The block matrices that correspond to pure linear accelerations and pure angular accelerations in the system (on the top left and on the bottom right) are symmetric. The matrix $\mathrm{C}$ contains coefficients of the first order derivatives of the generalized co-ordinates. Since the system is nonlinear, many elements of $\mathrm{C}$ contain first order derivatives of the generalized co-ordinates. The remaining terms in the dynamic equations resulting from gravitational potential energies and spring energies are collected in the matrix G. The coefficient matrices of the dynamic equations are given below,

Fcoeff $=\left[\begin{array}{cccccc}1 & 1 & \cos \left(\theta_{1}\right) & \cos \left(\theta_{1}\right) & \cos \left(\theta_{1}+\theta_{2}\right) & \cos \left(\theta_{1}+\theta_{2}\right) \\ 0 & 0 & 1 & 1 & \cos \left(\theta_{2}\right) & \cos \left(\theta_{2}\right) \\ 0 & 0 & 0 & 0 & 1 & 1 \\ 1 / 2 & -1 / 2 & 1 / 2 & -1 / 2 & 1 / 2+s_{2} \sin \left(\theta_{2}\right) & -1 / 2+s_{2} \sin \left(\theta_{2}\right) \\ 0 & 0 & 1 / 2 & -1 / 2 & 1 / 2 & -1 / 2 \\ 0 & 0 & 0 & 0 & 1 / 2 & -1 / 2\end{array}\right]$

$$
\begin{aligned}
& D(\underline{q})= \\
& {\left[\begin{array}{cccccc}
m_{1}+m_{2} & m_{2} \cos \left(\theta_{1}\right) & & & \\
+m_{3} & +m_{3} \cos \left(\theta_{1}\right) & m_{3} \cos \left(\theta_{1}+\theta_{2}\right) & \begin{array}{c}
-m_{2} \sin \left(\theta_{1}\right) \\
-m_{3} \sin \left(\theta_{1}\right)
\end{array} & -m_{3} s_{3} \sin \left(\theta_{1}+\theta_{2}\right) & 0 \\
& & & m_{3} s_{3} \sin \left(\theta_{1}+\theta_{2}\right) & \\
m_{2} \cos \left(\theta_{1}\right) & m_{2}+m_{3} & m_{3} \cos \left(\theta_{2}\right) & -m_{3} s_{3} \sin \left(\theta_{2}\right) & -m_{3} s_{3} \sin \left(\theta_{2}\right) & 0 \\
+m_{3} \cos \left(\theta_{1}\right) & & & 0 & 0 \\
m_{3} \cos \left(\theta_{1}+\theta_{2}\right) & m_{3} \cos \left(\theta_{2}\right) & m_{3} & m_{3} s_{3} \sin \left(\theta_{2}\right) & \\
-m_{2} s_{2} \sin \left(\theta_{1}\right) & & & m_{2} s_{2}^{2}+I_{1}+I_{2} & I_{2}+m_{3} s_{3}^{2}+I_{3} & I_{3} \\
-m_{3} s_{2} \sin \left(\theta_{1}\right) & -m_{3} s_{3} \sin \left(\theta_{2}\right) & m_{3} s_{2} \sin \left(\theta_{2}\right) & +I_{3}+m_{3} s_{2}^{2}+m_{3} s_{3}^{2} & +m_{3} s_{3} \cos \left(\theta_{2}\right) s_{2} & \\
-m_{3} s_{3} \sin \left(\theta_{1}+\theta_{2}\right) & & & +2 m_{3} s_{3} \cos \left(\theta_{2}\right) s_{2} & & \\
& & & I_{2}+m_{3} s_{3}^{2}+I_{3} & I_{2}+m_{3} s_{3}^{2}+I_{3} & I_{3} \\
-m_{3} s_{3} \sin \left(\theta_{1}+\theta_{2}\right) & -m_{3} s_{3} \sin \left(\theta_{2}\right) & 0 & +m_{3} s_{3} \cos \left(\theta_{2}\right) s_{2} I & & I_{3}
\end{array}\right]}
\end{aligned}
$$




$$
\begin{aligned}
& C(\underline{q})
\end{aligned}
$$

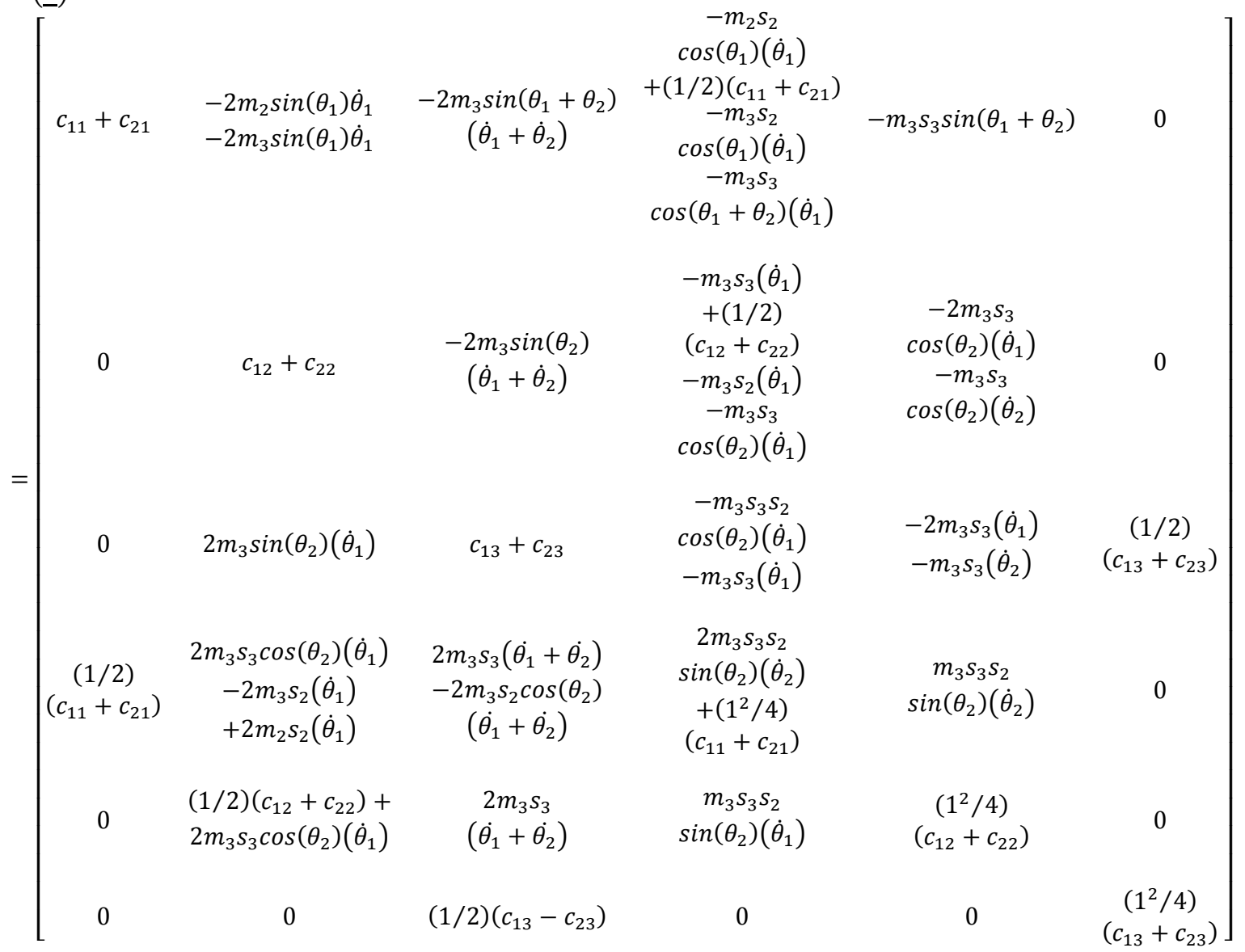

$$
G(\underline{q})=\left[\begin{array}{c}
-m_{1} g-m_{2} g+k_{11}\left(s_{1}+(1 / 2) \theta_{1}-s_{01}\right)+k_{21}\left(s_{1}-(1 / 2) \theta_{1}-s_{01}\right)-m_{3} g \\
-m_{2} g \cos \left(\theta_{1}\right)+k_{12}\left(s_{2}+(1 / 2) \theta_{2}-s_{02}\right)+k_{22}\left(s_{2}-(1 / 2) \theta_{2}-s_{02}\right)-m_{3} g \cos \left(\theta_{1}\right) \\
-m_{3} g \cos \left(\theta_{1}+\theta_{2}\right)+k_{13}\left(s_{3}+(1 / 2) \theta_{3}-s_{03}\right)+k_{23}\left(s_{3}-(1 / 2) \theta_{3}-s_{03}\right) \\
m_{2} s_{2} g \sin \left(\theta_{1}\right)+m_{3} s_{3} g \sin \left(\theta_{1}+\theta_{2}\right)+m_{3} s_{2} g \sin \left(\theta_{1}\right)+k_{11}\left(s_{1}+(1 / 2) \theta_{1}-s_{01}\right)(1 / 2) \\
+k_{21}\left(s_{1}-(1 / 2) \theta_{1}-s_{01}\right)(-1 / 2) \\
m_{3} s_{3} g \sin \left(\theta_{1}+\theta_{2}\right)+k_{12}\left(s_{2}+(1 / 2) \theta_{2}-s_{02}\right)(1 / 2)+k_{22}\left(s_{2}-(1 / 2) \theta_{2}-s_{02}\right)(-1 / 2) \\
k_{13}\left(s_{3}+(1 / 2) \theta_{3}-s_{03}\right)(1 / 2)+k_{23}\left(s_{3}-(1 / 2) \theta_{3}-s_{03}\right)(-1 / 2)
\end{array}\right]
$$




\subsection{Design Switching PD-plus Gravity Sliding Mode Controller}

A time-varying sliding surface $\boldsymbol{s}(\boldsymbol{x}, \boldsymbol{t})$ in the state space $\boldsymbol{R}^{\boldsymbol{n}}$ is given by [6]:

$s(x, t)=\left(\frac{d}{d t}+\lambda\right)^{n-1} \tilde{x}=0$

where $\lambda$ is the positive constant. To further penalize tracking error, integral part can be used in sliding surface part as follows [6]:

$s(x, t)=\left(\frac{d}{d t}+\lambda\right)^{n-1}\left(\int_{0}^{t} \tilde{x} d t\right)=0$

The main target in this methodology is kept the sliding surface slope $\boldsymbol{s}(\boldsymbol{x}, \boldsymbol{t})$ near to the zero. Therefore, one of the common strategies is to find input $\boldsymbol{U}$ outside of $\boldsymbol{s}(\boldsymbol{x}, \boldsymbol{t})$ [6].

$\frac{1}{2} \frac{d}{d t} s^{2}(x, t) \leq-\zeta|s(x, t)|$

where $\zeta$ is positive constant.

If $S(0)>0 \rightarrow \frac{d}{d t} S(t) \leq-\zeta$

if $S_{t_{\text {reach }}}=S(0) \rightarrow \operatorname{error}\left(x-x_{d}\right)=0$

suppose $\mathrm{S}$ is defined as

$s(x, t)=\left(\frac{d}{d t}+\lambda\right) \quad \tilde{x}=\left(\dot{x}-\dot{x}_{d}\right)+\lambda\left(x-x_{d}\right)$

The derivation of S, namely, $\dot{S}$ can be calculated as the following;

$\dot{S}=\left(\ddot{x}-\ddot{x}_{d}\right)+\lambda\left(\dot{x}-\dot{x}_{d}\right)$

suppose the second order system is defined as;

$\ddot{\mathrm{x}}=\mathrm{f}+\mathrm{u} \rightarrow \dot{\mathrm{S}}=\mathrm{f}+\mathrm{U}-\ddot{\mathrm{x}}_{\mathrm{d}}+\lambda\left(\dot{\mathrm{x}}-\dot{\mathrm{x}}_{\mathrm{d}}\right)$

Where $\boldsymbol{f}$ is the dynamic uncertain, and also since $S=0$ and $\dot{S}=0$, to have the best approximation, $\widehat{\boldsymbol{U}}$ is defined as

$\widehat{U}=-\hat{f}+\ddot{x}_{d}-\lambda\left(\dot{x}-\dot{x}_{d}\right)$ 
A simple solution to get the sliding condition when the dynamic parameters have uncertainty is the switching control law [52-53]:

$\mathrm{U}_{\mathrm{dis}}=\widehat{\mathrm{U}}-\mathrm{K}(\overrightarrow{\mathrm{x}}, \mathrm{t}) \cdot \operatorname{sgn}(\mathrm{s})$

where the switching function $\mathbf{s g n}(\mathbf{S})$ is defined as $[1,6]$

$\operatorname{sgn}(s)= \begin{cases}1 & s>0 \\ -1 & s<0 \\ 0 & s=0\end{cases}$

and the $\boldsymbol{K}(\overrightarrow{\boldsymbol{x}}, \boldsymbol{t})$ is the positive constant. Suppose by (17) the following equation can be written as,

$\frac{1}{2} \frac{d}{d t} s^{2}(x, t)=\dot{S} \cdot S=[f-\hat{f}-K \operatorname{sgn}(s)] \cdot S=(f-\hat{f}) \cdot S-K|S|$

and if the sliding surface can be calculated as

$s(x, t)=\left(\frac{d}{d t}+\lambda\right)^{2}\left(\int_{0}^{t} \tilde{x} d t\right)=\left(\dot{x}-\dot{x}_{d}\right)+2 \lambda\left(\dot{x}-\dot{x}_{d}\right)-\lambda^{2}\left(x-x_{d}\right)$

in this method the approximation of $\boldsymbol{U}$ is computed as [6]

$\widehat{U}=-\hat{f}+\ddot{x}_{d}-2 \lambda\left(\dot{x}-\dot{x}_{d}\right)+\lambda^{2}\left(x-x_{d}\right)$

Based on above discussion, the PD plus gravity based sliding mode control law for multi degrees of freedom

$\tau=G+\tau_{\text {dis }}$

continuum robot manipulator is written as $[1,6]$ :

Where, the discontinues component $\boldsymbol{\tau}_{\text {dis }}$ is computed as [1]:

$\tau_{\text {dis }}=K \cdot \operatorname{sgn}(S)$

the sliding mode control of robot manipulator is calculated as;

$\tau=G+K \cdot \operatorname{sgn}(S)$

where $S=\lambda e+\dot{e}$ in PD-SMC and $S=\lambda e+\dot{e}+\left(\frac{\lambda}{2}\right)^{2} \sum e$ in PID-SMC.

Figure 1 shows the application of PD + gravity in continuum robot manipulator. 


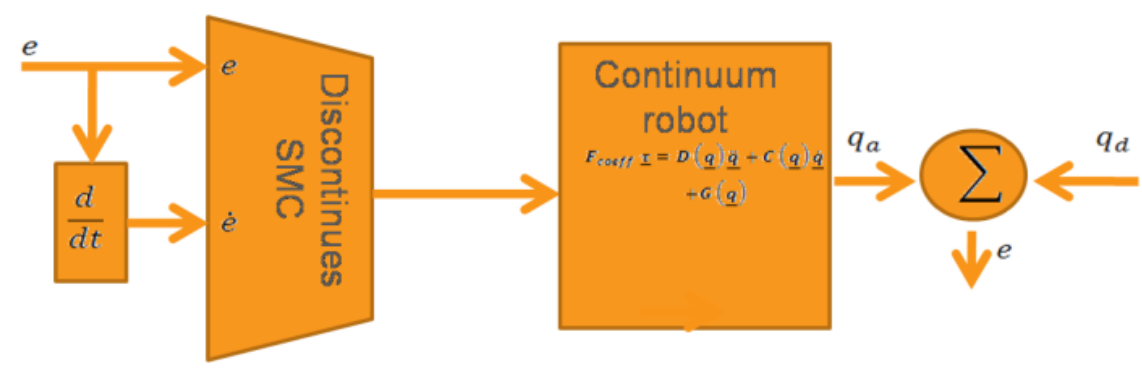

Fig 1: Block diagram of PD plus gravity sliding mode method with switching function

\subsection{Fuzzy Inference Engine:}

This section provides a review about foundation of fuzzy logic based on $[32,53]$. Supposed that $U$ is the universe of discourse and $x$ is the element of $U$, therefore, a crisp set can be defined as a set which consists of different elements $(x)$ will all or no membership in a set. A fuzzy set is a set that each element has a membership grade, therefore it can be written by the following definition;

$A=\left\{x, \mu_{A}(x) \mid x \in X\right\} ; A \in U$

Where an element of universe of discourse is $\mathrm{x}, \mu_{\mathrm{A}}$ is the membership function (MF) of fuzzy set. The membership function $\left(\mu_{\mathrm{A}}(\mathrm{x})\right)$ of fuzzy set $\mathrm{A}$ must have a value between zero and one. If the membership function $\mu_{\mathrm{A}}(\mathrm{x})$ value equal to zero or one, this set change to a crisp set but if it has a value between zero and one, it is a fuzzy set. Defining membership function for fuzzy sets has divided into two main groups; namely; numerical and functional method, which in numerical method each number has different degrees of membership function and functional method used standard functions in fuzzy sets. The membership function which is often used in practical applications includes triangular form, trapezoidal form, bell-shaped form, and Gaussian form.

Linguistic variable can open a wide area to use of fuzzy logic theory in many applications (e.g., control and system identification). In a natural artificial language all numbers replaced by words or sentences.

If - then Rule statements are used to formulate the condition statements in fuzzy logic. A single fuzzy If - then rule can be written by

If $x$ is $A$ Then $y$ is $B$

where $A$ and $B$ are the Linguistic values that can be defined by fuzzy set, the If - part of the part of " $x$ is $A$ " is called the antecedent part and the then - part of the part of " $y$ is $B$ " is called the Consequent or Conclusion part. The antecedent of a fuzzy if-then rule can have multiple parts, which the following rules shows the multiple antecedent rules:

if $e$ is $N B$ and $\dot{e}$ is $M L$ then $T$ is $L L$

where $e$ is error, $\dot{e}$ is change of error, $N B$ is Negative Big, $M L$ is Medium Left, $T$ is torque and $L L$ is Large Left. If - then rules have three parts, namely, fuzzify inputs, apply fuzzy operator and apply implication method which in fuzzify inputs the fuzzy statements in the antecedent replaced by the degree of membership, apply fuzzy operator used when the antecedent has multiple parts and replaced by single number between 0 to 
1, this part is a degree of support for the fuzzy rule, and apply implication method used in consequent of fuzzy rule to replaced by the degree of membership. The fuzzy inference engine offers a mechanism for transferring the rule base in fuzzy set which it is divided into two most important methods, namely, Mamdani method and Sugeno method. Mamdani method is one of the common fuzzy inference systems and he designed one of the first fuzzy controllers to control of system engine. Mamdani's fuzzy inference system is divided into four major steps: fuzzification, rule evaluation, aggregation of the rule outputs and defuzzification. Michio Sugeno use a singleton as a membership function of the rule consequent part. The following definition shows the Mamdani and Sugeno fuzzy rule base

$$
\begin{array}{llllc}
\text { Mamdani } & F . R^{1}: \text { if } & x \text { is } A \text { and } y \text { is } B & \text { then } & z \text { is } C \\
\text { Sugeno } & F . R^{1}: \text { if } & x \text { is } A \text { and } y \text { is } B & \text { then } & f(x, y) \text { is } C
\end{array}
$$

When $x$ and $y$ have crisp values fuzzification calculates the membership degrees for antecedent part. Rule evaluation focuses on fuzzy operation $(A N D / O R)$ in the antecedent of the fuzzy rules. The aggregation is used to calculate the output fuzzy set and several methodologies can be used in fuzzy logic controller aggregation, namely, Max-Min aggregation, Sum-Min aggregation, Max-bounded product, Max-drastic product, Max-bounded sum, Max-algebraic sum and Min-max. Two most common methods that used in fuzzy logic controllers are Max-min aggregation and Sum-min aggregation. Max-min aggregation defined as below

$\mu_{U}\left(x_{k}, y_{k}, U\right)=\mu_{\mathrm{U}_{i=1}^{r} F R^{i}}\left(x_{k}, y_{k}, U\right)=\max \left\{\min _{i=1}^{r}\left[\mu_{R_{p q}}\left(x_{k}, y_{k}\right), \mu_{p_{m}}(U)\right]\right\}$

The Sum-min aggregation defined as below

$$
\mu_{U}\left(x_{k}, y_{k}, U\right)=\mu_{\mathrm{U}_{i=1}^{r} F R^{i}}\left(x_{k}, y_{k}, U\right)=\sum \min _{i=1}^{r}\left[\mu_{R}\left(x_{k}, y_{k}\right), \mu_{p_{m}}(U)\right]
$$

where $r$ is the number of fuzzy rules activated by $x_{k}$ and $y_{k}$ and also $\mu_{\cup_{i=1}^{r} F R^{i}}\left(x_{k}, y_{k}, U\right)$ is a fuzzy interpretation of $i-t h$ rule. Defuzzification is the last step in the fuzzy inference system which it is used to transform fuzzy set to crisp set. Consequently defuzzification's input is the aggregate output and the defuzzification's output is a crisp number. Centre of gravity method (COG) and Centre of area method $(C O A)$ are two most common defuzzification methods, which COG method used the following equation to calculate the defuzzificatio

$\operatorname{COG}\left(x_{k}, y_{k}\right)=\frac{\sum_{i} U_{i} \sum_{j=1}^{r} \cdot \mu_{u}\left(x_{k}, y_{k}, U_{i}\right)}{\sum_{i} \sum_{j=1}^{r} \cdot \mu_{u}\left(x_{k}, y_{k}, U_{i}\right)}$

and $C O A$ method used the following equation to calculate the defuzzification

$\operatorname{COA}\left(x_{k}, y_{k}\right)=\frac{\sum_{i} U_{i} \cdot \mu_{u}\left(x_{k}, y_{k}, U_{i}\right)}{\sum_{i} \mu_{U} \cdot\left(x_{k}, y_{k}, U_{i}\right)}$

Where $\operatorname{COG}\left(x_{k}, y_{k}\right)$ and $\operatorname{COA}\left(x_{k}, y_{k}\right)$ illustrates the crisp value of defuzzification output, $U_{i} \in U$ is discrete element of an output of the fuzzy set, $\mu_{U}$. $\left(x_{k}, y_{k}, U_{i}\right)$ is the fuzzy set membership function, and $r$ is the number of fuzzy rules. 
Based on foundation of fuzzy logic methodology; fuzzy logic controller has played important rule to design nonlinear controller for nonlinear and uncertain systems [53]. However the application area for fuzzy control is really wide, the basic form for all command types of controllers consists of;

- Input fuzzification (binary-to-fuzzy[B/F]conversion)

- $\quad$ Fuzzy rule base (knowledge base)

- Inference engine

- Output defuzzification (fuzzy-to-binary[F/B]conversion).

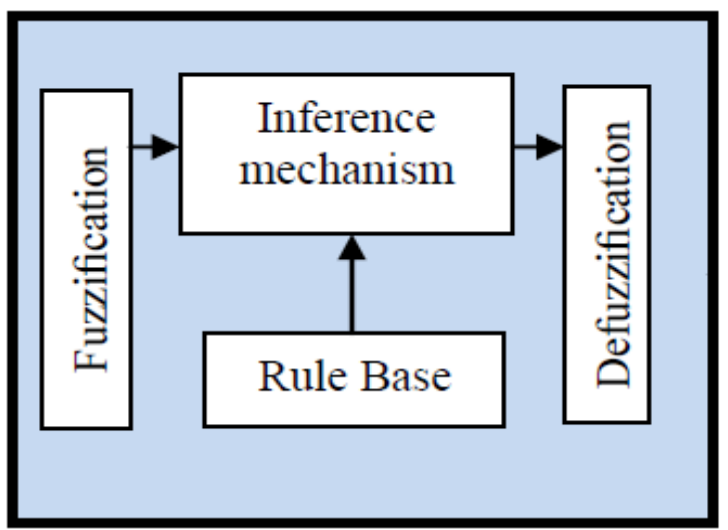

Fig 2: Block diagram of Fuzzy Inference System

\section{Methodology}

PD plus gravity switching fuzzy sliding mode controller is an important nonlinear controller in a partly uncertain dynamic system's parameters. This controller is used in several applications such as in robotics, process control, aerospace and power electronics. This controller is used to control of nonlinear dynamic systems particularly for continuum robot manipulators, because it has a suitable control performance and it is a robust and stable. Fuzzy logic theory is used in parallel with switching sliding PD plus gravity control to compensate the limited uncertainty in system's dynamic and reduces the chattering phenomenon. To achieve this goal, the dynamic equivalent part of pure sliding mode controller is modeled by Mamdani's performance/ error-based fuzzy logic methodology. Reduce or eliminate the chattering phenomenon and reduce the error are played important role, therefore switching method is used beside the artificial intelligence part to solve the chattering problem with respect to reduce the error. Robot manipulator's dynamic formulations are highly nonlinear and some of parameters are unknown therefore design a controller based on dynamic formulation is complicated. To solve this challenge parallel fuzzy logic methodology is applied to switching sliding mode PD plus gravity controller. In this method; error based Mamdani's fuzzy inference system has considered with two inputs, one output and totally 49 rules. Figure 3 shows error-based parallel fuzzy plus sliding mode controller. 


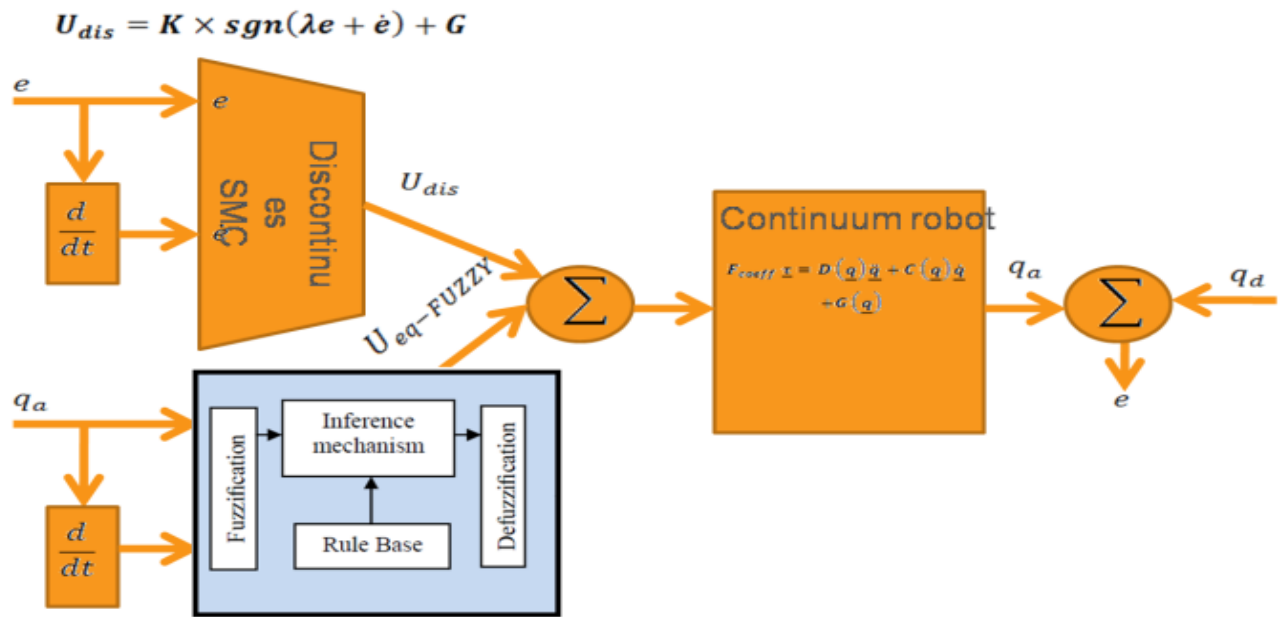

Fig 3: Block diagram of fuzzy inference system + PD plus gravity sliding mode method with switching function

For parallel fuzzy inference system plus PD plus gravity switching sliding mode controller the system performance is sensitive to the sliding surface slope coefficient $(\lambda)$. For instance, if large value of $\lambda$ is chosen the response is very fast the system is unstable and conversely, if small value of $\lambda$ is considered the response of system is very slow but system is stable. Therefore to have a good response, compute the best value sliding surface slope coefficient is very important. In parallel fuzzy inference system compensator of PD plus gravity switching sliding mode controller the PD-sliding surface is defined as follows:

$S=\dot{e}+\lambda e$

where $\lambda=\operatorname{diag}\left[\lambda_{11}, \lambda_{12}, \lambda_{13}\right]$. The time derivative of $S$ is computed;

$\dot{S}=\ddot{q}_{d}+\lambda \dot{e}$

Based on Figure 3, the output is written;

$\hat{\tau}=\tau_{e q_{f u z z y}}+\tau_{D I S+G R A V I T Y}$

Based on fuzzy logic methodology

$f(x)=U_{\text {fuzzy }}=\sum_{l=1}^{M} \theta^{T} \zeta(x)$

where $\boldsymbol{\theta}^{\boldsymbol{T}}$ is adjustable parameter (gain updating factor) and $\boldsymbol{\zeta}(\boldsymbol{x})$ is defined by;

$\zeta(x)=\frac{\sum_{i} \mu\left(x_{i}\right) x_{i}}{\sum_{i} \mu\left(x_{i}\right)}$ 
As mentioned in Figure 3, design an error-based parallel fuzzy compensate of equivalent part based on Mamdani's fuzzy inference method has four steps, namely, fuzzification, fuzzy rule base and rule evaluation, aggregation of the rule output (fuzzy inference system) and defuzzification.

Fuzzification: the first step in fuzzification is determine inputs and outputs which, it has two inputs $(e, \dot{e})$ and one output $\left(\tau_{f u z z y}\right)$. The inputs are error (e) which measures the difference between desired and actual output position, and the change of error $(\dot{e})$ which measures the difference between desired and actual velocity and output is fuzzy equivalent torque. The second step is chosen an appropriate membership function for inputs and output which, to simplicity in implementation because it is a linear function with regard to acceptable performance triangular membership function is selected in this research. The third step is chosen the correct labels for each fuzzy set which, in this research namely as linguistic variable. Based on experience knowledge the linguistic variables for error (e) are; Negative Big (NB), Negative Medium (NM), Negative Small (NS), Zero (Z), Positive Small (PS), Positive Medium (PM), Positive Big (PB), and based on literature [40] and experience knowledge it is quantized into thirteen levels represented by: $-1,-0.83,-0.66,-0.5,-0.33,-0.16,0$, $0.16,0.33,0.5,0.66,0.83,1$ the linguistic variables for change of error $(\dot{e})$ are; Fast Left (FL), Medium Left (ML), Slow Left (SL),Zero (Z), Slow Right (SR), Medium Right (MR), Fast Right (FR), and it is quantized in to thirteen levels represented by: $-6,-5,-0.4,-3,-2,-1,0,1,2,3,4,5,6$, and the linguistic variables to find the output are; Large Left (LL), Medium Left (ML), Small Left (SL), Zero (Z), Small Right (SR), Medium Right (MR), Large Right (LR) and it is quantized in to thirteen levels represented by: $-85,-70.8,-56.7,-42.5,-28.3$, $-14.2,0,14.2,28.3,42.5,56.7,70.8,85$.

Fuzzy rule base and rule evaluation: the first step in rule base and evaluation is to provide a least structured method to derive the fuzzy rule base which, expert experience and control engineering knowledge is used because this method is the least structure of the other one and the researcher derivation the fuzzy rule base from the knowledge of system operate and/or the classical controller. Design the rule base of fuzzy inference system can play important role to design the best performance of parallel fuzzy plus PD applied gravity switching sliding mode controller, that to calculate the fuzzy rule base the researcher is used to heuristic method which, it is based on the behavior of the control of continuum robot manipulator. The complete rule base for this controller is shown in Table 1. Rule evaluation focuses on operation in the antecedent of the fuzzy rules in fuzzy PD plus gravity switching sliding mode controller. This part is used $A N D / O R$ fuzzy operation in antecedent part which $A N D$ operation is used.

Aggregation of the rule output (Fuzzy inference): based onfuzzy methodology, Max-Min aggregation is used in this work.

Table 1. Modified Fuzzy rule base table

Decrease the overshoot
\begin{tabular}{|c|c|c|c|c|c|c|c|}
\hline$\dot{e}$ & FL & ML & SL & Z & SR & MR & FR \\
\hline NB & LL & LL & LL & ML & SL & SL & Z \\
\hline NM & LL & ML & ML & ML & SL & Z & SR \\
\hline NS & LL & ML & SL & SL & Z & SR & MR \\
\hline Z & LL & ML & SL & Z & SR & MR & LR \\
\hline PS & ML & SL & Z & SR & SR & MR & LR \\
\hline PM & SL & Z & SR & MR & MR & MR & LR \\
\hline PB & Z & SR & SR & MR & LR & LR & LR \\
\hline
\end{tabular}


Defuzzification: The last step to design fuzzy inference in our parallel fuzzy compensator PD plus gravity switching sliding mode controller is defuzzification. This part is used to transform fuzzy set to crisp set, therefore the input for defuzzification is the aggregate output and the output of it is a crisp number. Based on fuzzy methodology Center of Gravity method (COG) is used in this research. Table 2 shows the lookup table in parallel fuzzy compensator PD plus gravity switching sliding mode controller which is computed by COG defuzzification method. Table 2 has 169 cells to shows the error-based fuzzy compensate of equivalent part behavior.

Table 2. $\tau_{\text {fuzzy }}$ performance: lookup table in parallel fuzzy compensate of PD plus gravity switching sliding mode controller by COG

\begin{tabular}{|c|c|c|c|c|c|c|c|c|c|c|c|c|c|}
\hline$\dot{\boldsymbol{e}}$ & \multicolumn{13}{|c|}{ Membership Function ( $\tau_{\text {fuzzy }}$ ) } \\
\hline e & -6 & -5 & -4 & -3 & -2 & -1 & 0 & 1 & 2 & 3 & 4 & 5 & 6 \\
\hline-1 & -85 & -85 & -85 & -85 & -84 & -84 & -81 & -81 & -80 & -80 & -79 & -79 & -78 \\
\hline-0.83 & -80 & -80 & -80 & -78 & -75 & -70 & $\begin{array}{l}-70 \\
\end{array}$ & -65 & -64 & -60 & -56 & $\begin{array}{l}-54 \\
\end{array}$ & -47 \\
\hline-0.66 & -78 & -73 & -70 & -68 & -64 & -61 & -60 & -57 & -55 & -50 & -47 & -40 & -38 \\
\hline-0.5 & -70 & -60 & -58 & -51 & -42 & -38 & -34 & -33 & -31 & -29 & -28.4 & -28.1 & -28 \\
\hline-0.33 & -50 & -48 & -45 & -40 & -38 & -34 & -32 & -30 & -28 & -26 & -25 & -21 & -20 \\
\hline-0.16 & -30 & -25 & -21 & -18 & -16 & -14 & -10 & -9 & -8 & -7 & -6.8 & -6 & -5 \\
\hline $\mathbf{0}$ & -10 & -8 & -6 & -1 & 2 & 3 & 6 & 7 & 8 & 10 & 12 & 15 & 17 \\
\hline 0.16 & 15 & 18 & 21 & 22 & 23 & 25 & 27 & 28 & 29 & 30 & 30.5 & 30.8 & 31 \\
\hline $\mathbf{0 . 3 3}$ & 29 & 29.8 & 31 & 33 & 34 & 34.6 & 35 & 35.2 & 36 & 37 & 38 & 39 & 42 \\
\hline 0.5 & 40 & 41 & 42 & 43 & 45 & 45 & 46 & 46.3 & 46.8 & 47 & 48 & 51 & 52 \\
\hline 0.66 & 48 & 49 & 50 & 52 & 53 & 55 & 56 & 57 & 58 & 59 & 60 & 61 & 63 \\
\hline 0.83 & 60 & 61 & 62 & 63 & 64 & 66 & 67 & 68 & 68.5 & 69 & 70 & 70.8 & 71 \\
\hline 1 & 74 & 75 & 75 & 78 & 78 & 78 & 80 & 80 & 85 & 85 & 85 & 85 & 85 \\
\hline
\end{tabular}

\section{Results and discussion}

Linear PD controller (PD), PD plus gravity and proposed PD fuzzy PD plus gravity switching sliding mode methodology were tested to step response trajectory. The simulation was implemented in MATLAB/SIMULINK environment. Position trajectory and disturbance rejection are compared in these controllers.

Position trajectory: Figure 4 shows the links trajectory in these three methodologies without disturbance for step trajectory in general and zoom scaling. 

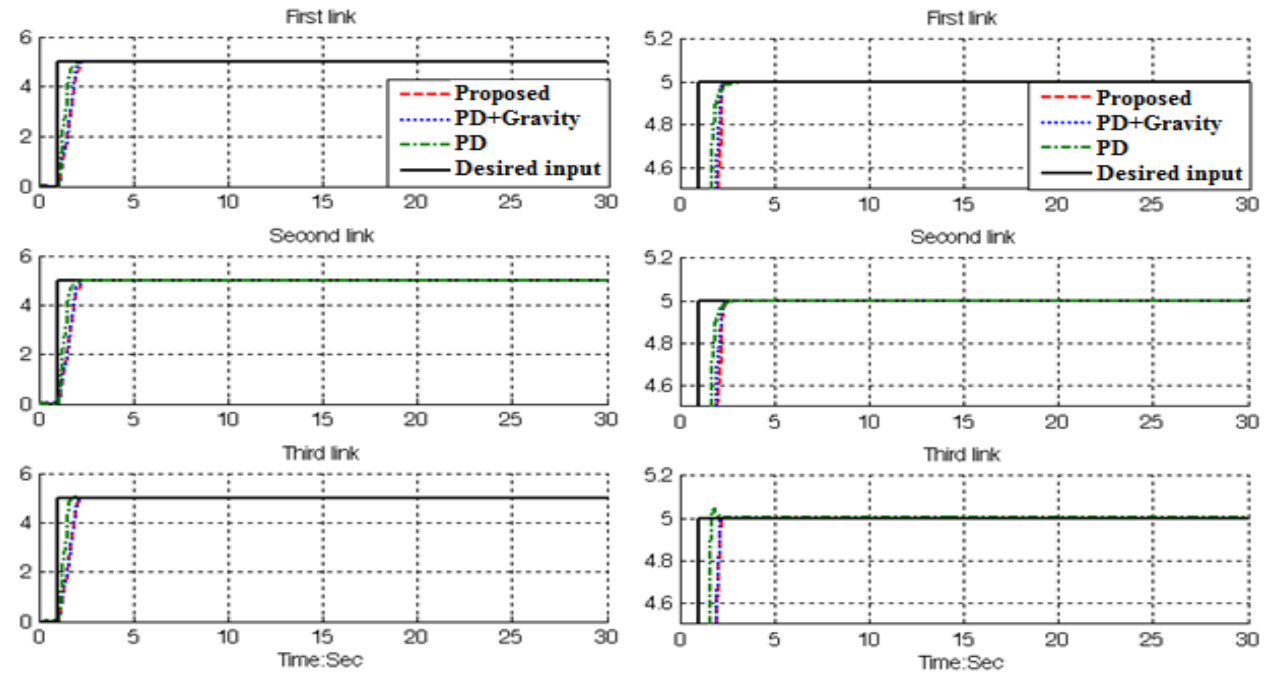

Fig 4: PD controller, PD plus gravity controller and proposed method without disturbance

Based on Figure 4 it is observed that, the overshoot in proposed method is $0 \%$, in PD's is $1 \%$ and in PD plus gravity's is $0 \%$, and rise time in proposed method's is 0.6 seconds, in PD's is 0.483 second and in PD plus gravity's is about 0.6 seconds. From the trajectory MATLAB simulation for proposed method, PD and PD plus gravity in certain system, it was seen that all of three controllers have acceptable performance.

Disturbance rejection: Figure 5 is indicated the power disturbance removal in PD controller, PD+gravity controller and proposed method. Besides a band limited white noise with predefined of $40 \%$ the power of input signal is applied to the step trajectory; it found slight oscillations in PD and PD+gravity position responses.
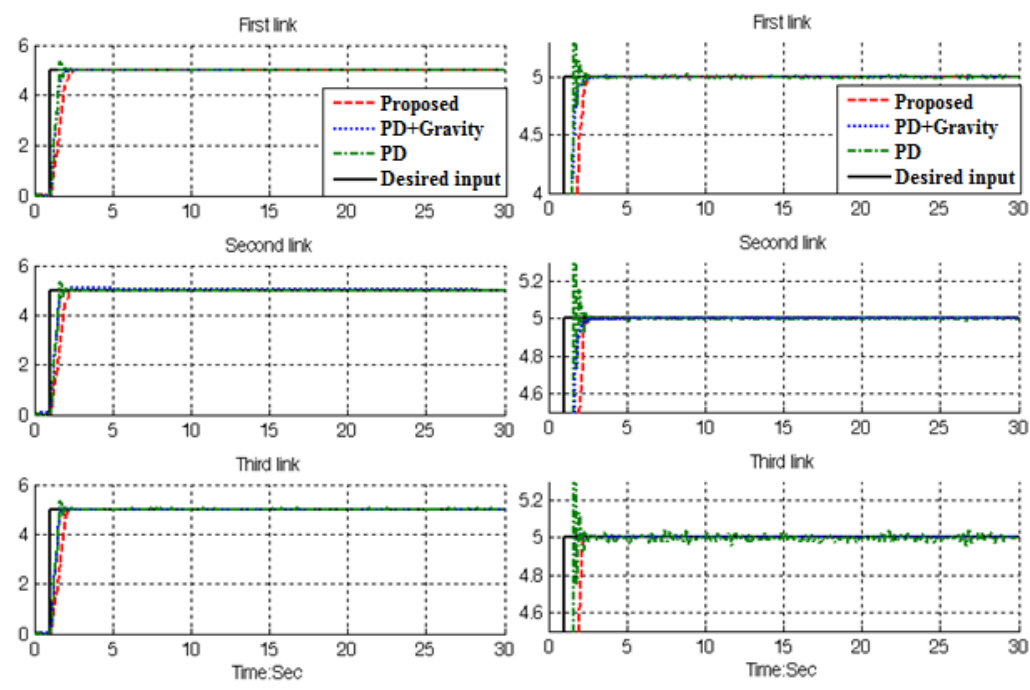

Fig 5: PD, PD+gravity and Proposed method: Continuum robot arm with external disturbance 
Based on Figure 5; by comparing step response trajectory with $40 \%$ disturbance of relative to the input signal amplitude in proposed method, PD plus gravity and PD controller, proposed method's overshoot about $(0 \%)$ is lower than PD + gravity's $(0.5 \%)$ and PD controller's $(1 \%)$. PD controller's rise time $(0.5$ seconds $)$ is lower than PD + gravity's ( 0.63 second $)$ and proposed method's $(0.65$ second $)$.

\section{Conclusion}

Refer to this research, a position fuzzy-based tuning PD plus gravity switching sliding mode controller is proposed for continuum robot manipulator. The main problem in pure sliding mode controller with switching function was chattering phenomenon in certain and uncertain systems. The chattering phenomenon problem can be reduced in certain and partly system by using fuzzy logic methodology in PD plus gravity switching sliding mode technique law. The simulation results exhibit that the PD plus gravity with switching sliding mode controller works well in certain system. The nonlinear equivalent dynamic problem in uncertain system is solved by using fuzzy logic theory. To eliminate the continuum robot manipulator system's dynamic, 49 rules Mamdani inference system is design and applied to PD plus gravity switching sliding mode methodology. This methodology is based on applied fuzzy logic in equivalent nonlinear dynamic part to estimate unknown parameters. The results demonstrate that the parallel error-based fuzzy PD plus gravity switching sliding mode controller is a model-free controllers which works well in certain and partly uncertain system. Based on theoretical and simulation results, it is observed that parallel fuzzy-based tuning PD plus gravity switching sliding mode controller is a model-free stable control for continuum robot manipulator. It is a best solution to eliminate chattering phenomenon with switching function in structure and unstructured uncertainties.

\section{ACKNOWLEDGMENT}

The authors would like to thank the anonymous reviewers for their careful reading of this paper and for their helpful comments. This work was supported by the SSP Research and Development Corporation Program of Iran under grant no. 20132014-Persian Gulf-1A.

\section{REFERENCES:}

[1] T. R. Kurfess, Robotics and automation handbook: CRC, 2005.

[2] J. J. E. Slotine and W. Li, Applied nonlinear control vol. 461: Prentice hall Englewood Cliffs, NJ, 1991.

[3] K. Ogata, Modern control engineering: Prentice Hall, 2009.

[4] J. J. D'Azzo, C. H. Houpis and S. N. Sheldon, Linear control system analysis and design with MATLAB: CRC, 2003.

[5] B. Siciliano and O. Khatib, Springer handbook of robotics: Springer-Verlag New York Inc, 2008.

[6] F. T. Cheng, T. L. Hour, Y. Y. Sun and T. H. Chen, "Study and resolution of singularities for a 6-DOF PUMA manipulator," Systems, Man, and Cybernetics, Part B: Cybernetics, IEEE Transactions on, No. 2, vol. 27, pp. 332-343, 2002.

[7] M. W. Spong and M. Vidyasagar, Robot dynamics and control: Wiley-India, 2009.

[8] Farzin Piltan and Shahnaz Tayebi Haghighi, "Design Gradient Descent Optimal Sliding Mode Control of Continuum Robot, IAES-International Journal of Robotics and Automation, No.4, Vol. 1, pp. 175189 , 
[9] M. Bazregar, Farzin Piltan, A. Nabaee and M. Ebrahimi, " Parallel Soft Computing Control Optimization Algorithm for Uncertainty Dynamic Systems," International Journal of Advanced Science and Technology (IJAST), Vol. 51, pp. 93-106, 2013.

[10] Farzin Piltan , N. Sulaiman, Zahra Tajpaykar, Payman Ferdosali, Mehdi Rashidi, "Design Artificial Nonlinear Robust Controller Based on CTLC and FSMC with Tunable Gain," International Journal of Robotic and Automation, 2 (3): 205-220, 2011.

[11] Farzin Piltan, A. R. Salehi and Nasri B Sulaiman.," Design artificial robust control of second order system based on adaptive fuzzy gain scheduling," world applied science journal (WASJ), 13 (5): 10851092, 2011.

[12] Farzin Piltan, N. Sulaiman, Atefeh Gavahian, Samira Soltani, Samaneh Roosta, "Design Mathematical Tunable Gain PID-Like Sliding Mode Fuzzy Controller with Minimum Rule Base," International Journal of Robotic and Automation, 2 (3): 146-156, 2011.

[13] Farzin Piltan , M. Akbari, M. Piran, M. Bazrega "Design Model Free Switching Gain Scheduling Baseline Controller with Application to Automotive Engine," International Journal of Information Technology and Computer Science (IJITCS), 5 (1): 65-76, 2013.

[14] Farzin Piltan , M. Piran, M. Bazregar, M. Akbari "Design High Impact Fuzzy Baseline Variable Structure Methodology to Artificial Adjust Fuel Ratio ," International Journal of Intelligent Systems and Applications(IJISA), 5 (2): 59-70, 2013.

[15] Farzin Piltan, N. Sulaiman , Arash Zargari, Mohammad Keshavarz, Ali Badri , "Design PID-Like Fuzzy Controller With Minimum Rule Base and Mathematical Proposed On-line Tunable Gain: Applied to Robot Manipulator," International Journal of Artificial intelligence and expert system, 2 (4):184-195, 2011.

[16] Farzin Piltan, Nasri Sulaiman, M. H. Marhaban and R. Ramli, "Design On-Line Tunable Gain Artificial Nonlinear Controller,” Journal of Advances In Computer Research, 2 (4): 75-83, 2011.

[17] Farzin Piltan, N. Sulaiman, Payman Ferdosali, Iraj Assadi Talooki, "Design Model Free Fuzzy Sliding Mode Control: Applied to Internal Combustion Engine," International Journal of Engineering, 5 (4):302312, 2011.

[18] Farzin Piltan, N. Sulaiman, Samaneh Roosta, M.H. Marhaban, R. Ramli, "Design a New Sliding Mode Adaptive Hybrid Fuzzy Controller,” Journal of Advanced Science \& Engineering Research , 1 (1): 115 123, 2011.

[19] Farzin Piltan, Atefe Gavahian, N. Sulaiman, M.H. Marhaban, R. Ramli, "Novel Sliding Mode Controller for robot manipulator using FPGA," Journal of Advanced Science \& Engineering Research, 1 (1): 1-22, 2011.

[20] Farzin Piltan, N. Sulaiman, A. Jalali \& F. Danesh Narouei, "Design of Model Free Adaptive Fuzzy Computed Torque Controller: Applied to Nonlinear Second Order System," International Journal of Robotics and Automation, 2 (4):232-244, 2011.

[21] Farzin Piltan, N. Sulaiman, Iraj Asadi Talooki, Payman Ferdosali, "Control of IC Engine: Design a Novel MIMO Fuzzy Backstepping Adaptive Based Fuzzy Estimator Variable Structure Control ," International Journal of Robotics and Automation, 2 (5):360-380, 2011.

[22] Farzin Piltan, N. Sulaiman, Payman Ferdosali, Mehdi Rashidi, Zahra Tajpeikar, "Adaptive MIMO Fuzzy Compensate Fuzzy Sliding Mode Algorithm: Applied to Second Order Nonlinear System," International Journal of Engineering, 5 (5): 380-398, 2011.

[23] Farzin Piltan, N. Sulaiman, Hajar Nasiri, Sadeq Allahdadi, Mohammad A. Bairami, "Novel Robot Manipulator Adaptive Artificial Control: Design a Novel SISO Adaptive Fuzzy Sliding Algorithm Inverse Dynamic Like Method,” International Journal of Engineering, 5 (5): 399-418, 2011.

[24] Samira Soltani \& Farzin Piltan, "Design Artificial Nonlinear Controller Based on Computed Torque like Controller with Tunable Gain”. World Applied Science Journal, 14 (9): 1306-1312, 2011. 
[25] Farzin Piltan, N. Sulaiman, Sadeq Allahdadi, Mohammadali Dialame, Abbas Zare, "Position Control of Robot Manipulator: Design a Novel SISO Adaptive Sliding Mode Fuzzy PD Fuzzy Sliding Mode Control," International Journal of Artificial intelligence and Expert System, 2 (5):208-228, 2011.

[26] Farzin Piltan, SH. Tayebi HAGHIGHI, N. Sulaiman, Iman Nazari, Sobhan Siamak, "Artificial Control of PUMA Robot Manipulator: A-Review of Fuzzy Inference Engine And Application to Classical Controller ," International Journal of Robotics and Automation, 2 (5):401-425, 2011.

[27] Farzin Piltan, N. Sulaiman, Abbas Zare, Sadeq Allahdadi, Mohammadali Dialame, "Design Adaptive Fuzzy Inference Sliding Mode Algorithm: Applied to Robot Arm," International Journal of Robotics and Automation , 2 (5): 283-297, 2011.

[28] Farzin Piltan, Amin Jalali, N. Sulaiman, Atefeh Gavahian, Sobhan Siamak, "Novel Artificial Control of Nonlinear Uncertain System: Design a Novel Modified PSO SISO Lyapunov Based Fuzzy Sliding Mode Algorithm ," International Journal of Robotics and Automation, 2 (5): 298-316, 2011.

[29] Farzin Piltan, N. Sulaiman, Amin Jalali, Koorosh Aslansefat, "Evolutionary Design of Mathematical tunable FPGA Based MIMO Fuzzy Estimator Sliding Mode Based Lyapunov Algorithm: Applied to Robot Manipulator,” International Journal of Robotics and Automation, 2 (5):317-343, 2011.

[30] Farzin Piltan, N. Sulaiman, Samaneh Roosta, Atefeh Gavahian, Samira Soltani, "Evolutionary Design of Backstepping Artificial Sliding Mode Based Position Algorithm: Applied to Robot Manipulator," International Journal of Engineering, 5 (5):419-434, 2011.

[31] Farzin Piltan, N. Sulaiman, S.Soltani, M. H. Marhaban \& R. Ramli, "An Adaptive sliding surface slope adjustment in PD Sliding Mode Fuzzy Control for Robot Manipulator," International Journal of Control and Automation , 4 (3): 65-76, 2011.

[32] Farzin Piltan, N. Sulaiman, Mehdi Rashidi, Zahra Tajpaikar, Payman Ferdosali, "Design and Implementation of Sliding Mode Algorithm: Applied to Robot Manipulator-A Review ," International Journal of Robotics and Automation, 2 (5):265-282, 2011.

[33] Farzin Piltan, N. Sulaiman, Amin Jalali, Sobhan Siamak, and Iman Nazari, "Control of Robot Manipulator: Design a Novel Tuning MIMO Fuzzy Backstepping Adaptive Based Fuzzy Estimator Variable Structure Control ,” International Journal of Control and Automation, 4 (4):91-110, 2011.

[34] Farzin Piltan, N. Sulaiman, Atefeh Gavahian, Samaneh Roosta, Samira Soltani, "On line Tuning Premise and Consequence FIS: Design Fuzzy Adaptive Fuzzy Sliding Mode Controller Based on Lyaponuv Theory," International Journal of Robotics and Automation, 2 (5):381-400, 2011.

[35] Farzin Piltan, N. Sulaiman, Samaneh Roosta, Atefeh Gavahian, Samira Soltani, "Artificial Chattering Free on-line Fuzzy Sliding Mode Algorithm for Uncertain System: Applied in Robot Manipulator," International Journal of Engineering, 5 (5):360-379, 2011.

[36] Farzin Piltan, N. Sulaiman and I.AsadiTalooki, "Evolutionary Design on-line Sliding Fuzzy Gain Scheduling Sliding Mode Algorithm: Applied to Internal Combustion Engine," International Journal of Engineering Science and Technology, 3 (10):7301-7308, 2011.

[37] Farzin Piltan, Nasri B Sulaiman, Iraj Asadi Talooki and Payman Ferdosali.," Designing On-Line Tunable Gain Fuzzy Sliding Mode Controller Using Sliding Mode Fuzzy Algorithm: Applied to Internal Combustion Engine," world applied science journal (WASJ), 15 (3): 422-428, 2011.

[38] B. K. Yoo and W. C. Ham, "Adaptive control of robot manipulator using fuzzy compensator," Fuzzy Systems, IEEE Transactions on, No. 2, vol. 8, pp. 186-199, 2002.

[39] Y. S. Kung, C. S. Chen and G. S. Shu, "Design and Implementation of a Servo System for Robotic Manipulator," CACS, 2005.

[40] Farzin Piltan, N. Sulaiman, M. H. Marhaban, Adel Nowzary, Mostafa Tohidian," "Design of FPGA based sliding mode controller for robot manipulator," International Journal of Robotic and Automation, 2 (3): 183-204, 2011.

[41] Farzin Piltan, M. Mirzaie, F. Shahriyari, Iman Nazari \& S. Emamzadeh.” Design Baseline Computed Torque Controller" International Journal of Engineering, 3(3): 2012. 
[42] Farzin Piltan, H. Rezaie, B. Boroomand, Arman Jahed," Design robust back stepping online tuning feedback linearization control applied to IC engine," International Journal of Advance Science and Technology, 42: 183-204, 2012.

[43] Farzin Piltan, I. Nazari, S. Siamak, P. Ferdosali ,'Methodology of FPGA-based mathematical errorbased tuning sliding mode controller" International Journal of Control and Automation, 5(1): 89-110, 2012.

[44] Farzin Piltan, M. A. Dialame, A. Zare, A. Badri ,’Design Novel Lookup table changed Auto Tuning FSMC: Applied to Robot Manipulator" International Journal of Engineering, 6(1): 25-40, 2012.

[45] Farzin Piltan, B. Boroomand, A. Jahed, H. Rezaie ,"Methodology of Mathematical Error-Based Tuning Sliding Mode Controller" International Journal of Engineering, 6(2): 96-112, 2012.

[46] Farzin Piltan, F. Aghayari, M. R. Rashidian, M. Shamsodini, "A New Estimate Sliding Mode Fuzzy Controller for Robotic Manipulator" International Journal of Robotics and Automation, 3(1): 45-58, 2012.

[47] Farzin Piltan, M. Keshavarz, A. Badri, A. Zargari, "Design novel nonlinear controller applied to robot manipulator: design new feedback linearization fuzzy controller with minimum rule base tuning method" International Journal of Robotics and Automation, 3(1): 1-18, 2012.

[48] Piltan, F., et al. "Design sliding mode controller for robot manipulator with artificial tunable gain". Canaidian Journal of pure and applied science, 5 (2), 1573-1579, 2011.

[49] Farzin Piltan, A. Hosainpour, E. Mazlomian, M.Shamsodini, M.H Yarmahmoudi. "Online Tuning Chattering Free Sliding Mode Fuzzy Control Design: Lyapunov Approach" International Journal of Robotics and Automation, 3(3): 2012.

[50] Farzin Piltan, M.H. Yarmahmoudi, M. Shamsodini, E.Mazlomian, A.Hosainpour. " PUMA-560 Robot Manipulator Position Computed Torque Control Methods Using MATLAB/SIMULINK and Their Integration into Graduate Nonlinear Control and MATLAB Courses" International Journal of Robotics and Automation, 3(3): 2012.

[51] Farzin Piltan, R. Bayat, F. Aghayari, B. Boroomand. "Design Error-Based Linear Model-Free Evaluation Performance Computed Torque Controller" International Journal of Robotics and Automation, 3(3): 2012.

[52] Farzin Piltan, S. Emamzadeh, Z. Hivand, F. Shahriyari \& Mina Mirazaei . "PUMA-560 Robot Manipulator Position Sliding Mode Control Methods Using MATLAB/SIMULINK and Their Integration into Graduate/Undergraduate Nonlinear Control, Robotics and MATLAB Courses" International Journal of Robotics and Automation, 3(3): 2012.

[53] Farzin Piltan, J. Meigolinedjad, S. Mehrara, S. Rahmdel. " Evaluation Performance of $2^{\text {nd }}$ Order Nonlinear System: Baseline Control Tunable Gain Sliding Mode Methodology" International Journal of Robotics and Automation, 3(3): 2012.

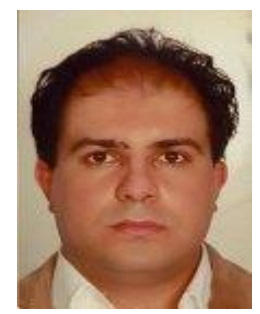

Farzin Piltan was born on 1975, Shiraz, Iran. In 2004 he is jointed the research and development company, SSP Co, Shiraz, Iran. In addition to 7 textbooks, Farzin Piltan is the main author of more than 70 scientific papers in refereed journals. He is editorial board of international journal of control and automation (IJCA), editorial board of International Journal of Intelligent System and Applications (IJISA), editorial board of IAES international journal of robotics and automation, editorial board of International Journal of Reconfigurable and Embedded Systems and reviewer of (CSC) international journal of robotics and automation. His main areas of research interests are nonlinear control, artificial control system and applied to FPGA, robotics and artificial nonlinear control and IC engine modelling and control. 

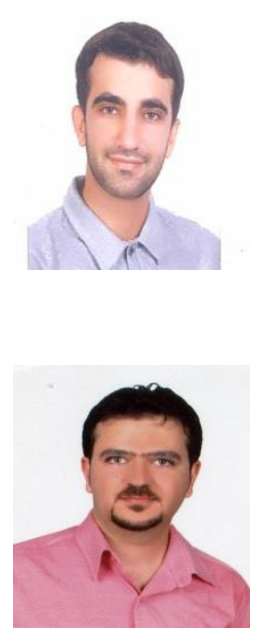

Mahdi Jafari is a communication and electrical engineer researcher at research and development company SSP. Co. He is now pursuing his Master in communication engineering at Shiraz University. His research activities deal with the robotics and artificial nonlinear control.

Mehdi Eram is an electrical engineer researcher at research and development company SSP. Co. His research activities deal with the robotics and artificial nonlinear control.

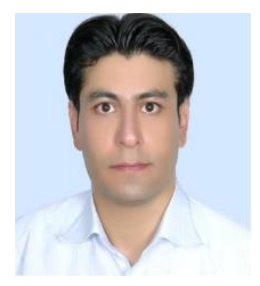

Omid Mahmoudi is an electrical and control researcher of research and development company SSP. Co. He is now pursuing his Master in control engineering at Shiraz university. His main areas are nonlinear control, artificial control system and robotics.

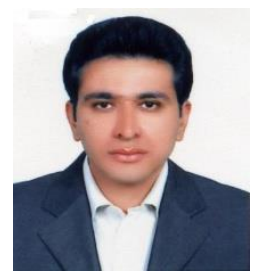

Omid Reza Sadrnia is a communication and electrical engineer researcher at research and development company SSP. Co. He is now pursuing his Master in communication engineering at Shiraz University. His research activities deal with the robotics and artificial nonlinear control.

How to cite this paper: Farzin Piltan,Mahdi Jafari,Mehdi Eram,Omid Mahmoudi,Omid Reza Sadrnia,"Design Artificial Intelligence-Based Switching PD plus Gravity for Highly Nonlinear Second Order System", IJEM, vol.3, no.1, pp.38-57, 2013.DOI: 10.5815/ijem.2013.01.04 